\title{
Designing a Collaborative Virtual Reality Game for Teen-Robot Interactions
}

\author{
Ada S. Kim \\ University of Washington \\ Seattle, Washington \\ kimsk@uw.edu \\ Simran Bhatia \\ University of Washington \\ Seattle, Washington \\ simran18@uw.edu
}

\author{
Elin A. Björling \\ University of Washington \\ Seattle, Washington \\ bjorling@uw.edu \\ Dong Li \\ University of Washington \\ Seattle, Washington \\ dongli@uw.edu
}

\section{ABSTRACT}

Virtual reality (VR) offers potential as a prototyping tool for human-robot interaction. We explored a way to utilize human-centered design (HCD) methodology to develop a collaborative VR game for understanding teens' perceptions of, and interactions with, social robots. Our paper features three stages of the design process for teen-robot interaction in VR; ideation, prototyping, and the game development. In the ideation stage, we identified three important design principles: collaboration, customization, and robot characterization. In the prototyping stage, we developed a card game, conducted gameplay, and confirmed our design principles. Finally, we developed a low-fidelity VR

Permission to make digital or hard copies of all or part of this work for personal or classroom use is granted without fee provided that copies are not made or distributed for profit or commercial advantage and that copies bear this notice and the full citation on the first page. Copyrights for components of this work owned by others than ACM must be honored. Abstracting with credit is permitted. To copy otherwise, or republish, to post on servers or to redistribute to lists, requires prior specific permission and/or a fee. Request permissions from permissions@acm.org.

IDC, May 2019, Boise, US

C 2019 Association for Computing Machinery.

ACM ISBN 978-x-xxxx-xxxx-x/YY/MM...\$15.00

DOI: $10.1145 / 3311927.3325314$ 
game and received teens' feedback. This exploratory study highlights the potential of VR, both for collaborative robot design and teen-robot interaction studies.

\section{CCS CONCEPTS}

- Human-centered computing $\rightarrow$ User studies; Virtual reality; Collaborative interaction; Usability testing; Field studies;

\section{KEYWORDS}

human-centered design, social robot, virtual reality, teens

\section{INTRODUCTION}

Despite numerous benefits identified from exploring human-robot interaction [2, 5], teenagers have been relatively understudied in the domain. A few previous studies, however, have shown many advantages of engaging teens in design processes [4, 14]. Using a participatory, human-centered design (HCD) approach, our study explored the human-robot interaction involving teens, especially focusing on the potential of Virtual reality (VR) platform [3, 12].

We collaborated with teens in two local high school clubs as co-designers to develop a VR game for teen-robot interaction. During a six-month period, we conducted three design stages: ideation, prototyping, and game development. In the first stage, we identified three important design principles: collaboration, characterization, and customization. Then we confirmed the principles and articulated detailed requirements for each principle, by developing and playing a card game prototype with teens. Finally, we developed a low-fidelity game by implementing the requirements to observe teen-robot interactions.

\section{RELATED WORK}

Figure 1: A summary table of design studies.

VR is an emerging new technology which holds great potential in the domain of robotics. A VR environment creates a sensation in which individuals perceive themselves as embodied in a virtual environment $[3,12]$. VR can accommodate a greater level of flexibility in one's sensorymotor experiences including reduced physical constraints and customizable 3D environments [1,7], which would benefit a design process. In robotics, it is complex and cost-intensive to physically create and test a mock design of a robot in the real world. In that sense, VR makes a useful platform for accelerating the design process by providing a flexible environment for building, prototyping, and testing a robot.

With its highly immersive, situated environment that creates embodied experiences, VR also has strong potential to support collaboration. For smooth collaboration, non-verbal cues such as gesture, eye gaze, or posture becomes important because they convey a large amount of socia information $[9,11]$. The social realism that VR environment provides is enough to incorporate such 

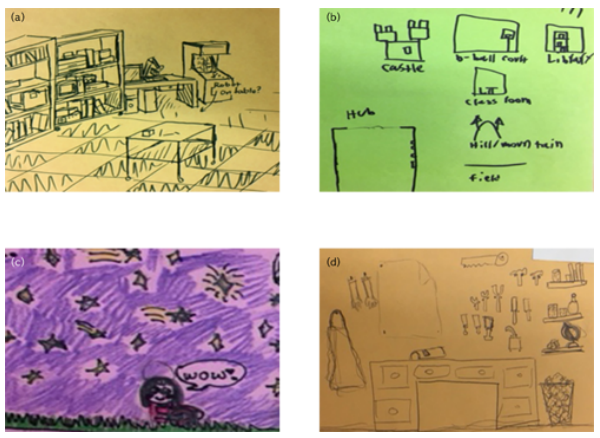

Figure 2: Teen's drawing of virtual design environments.

Teens articulated their preferred environments for the design of a robot in virtual reality.

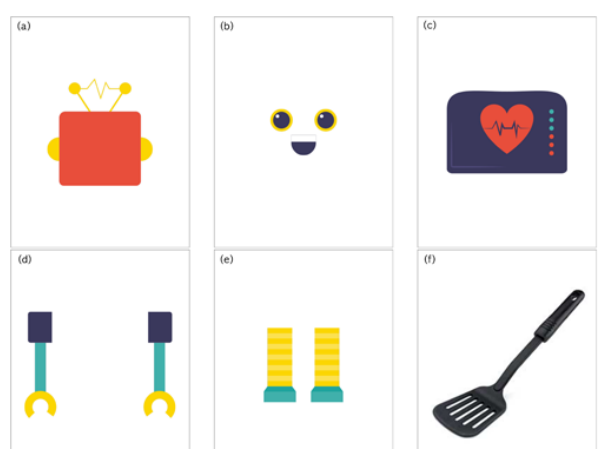

Figure 3: Samples of each robot part in the card.

The robot part cards consist of six categories: head, facial expression, body, arms, legs, and other accessories. non-verbal human expressions and enables real-time social interactions [13], which becomes ground for collaboration among multiple people.

There are two forms of collaboration in VR. One is asymmetric [6], meaning a collaboration between a person outside VR and the other person inside VR. The outside person sitting at a desktop computer, observes or instructs the other person who is immersed inside VR. The other is symmetric [8, 10] allowing multiple people to interact within a shared VR environment and perform tasks together.

\section{DESIGN STUDY}

For the study, we engaged teens from clubs at two local high schools as co-designers for six months. We conducted three design stages: ideation, prototyping, and VR game development. We have to note that teens' participation for design sessions was voluntary and varied depending on their availability. The number of participants in each design stage are indicated in table (Figure 1).

\section{Design Stage 1: Ideation (Brainstorming and Sketching)}

The first design stage was an exploration with teens about an appropriate environment for robot design. Participants were 12 students (ranging in age from 14 to 17) from a club. During a 45-minute participatory design session, we asked teens to freely describe their ideal robot-building environment and robot design. To encourage the brainstorming, we used a sticky note technique.

Teens provided detailed drawings and verbal articulations of environment designs including common spaces such as a classroom or a library (Figure 2). For robot part design, they suggested a modularized system in which different robot parts (e.g., heads, arms, legs, etc.) could be added, removed, or exchanged for customization. Teens responded to the idea of designing a robot together as "interesting," and "pretty great." They also expressed a desire to have a "truly collaborative" environment in which they felt connected to one another and worked together to design a robot. Finally, teens also shared what they felt were appropriate robot attributes (e.g., being a good listener, helpful, etc.)

This session informed us three design principles for creating a VR environment. Customization became the first principle of our design process as teens wanted to have a wide range of selections for customizing social robots to meet their preferences. Characterization of robot became the second principle; many of teens suggested their idea of an 'ideal character' of robot with which they wanted to interact. Collaboration became the last principle as teens expressed highly positive attitude toward the idea of collaborating with their peers for building a social robot.

\section{Design Stage 2: Prototyping a Card Game}

Inspired by the Robot Design Game (http://robot-design.org/), we designed a card game as a preliminary prototype of the VR game. The card deck included six environment cards and several robot parts such as faces, bodies, accessories, etc. (Figure 3). For characterization, we added robot attribute 

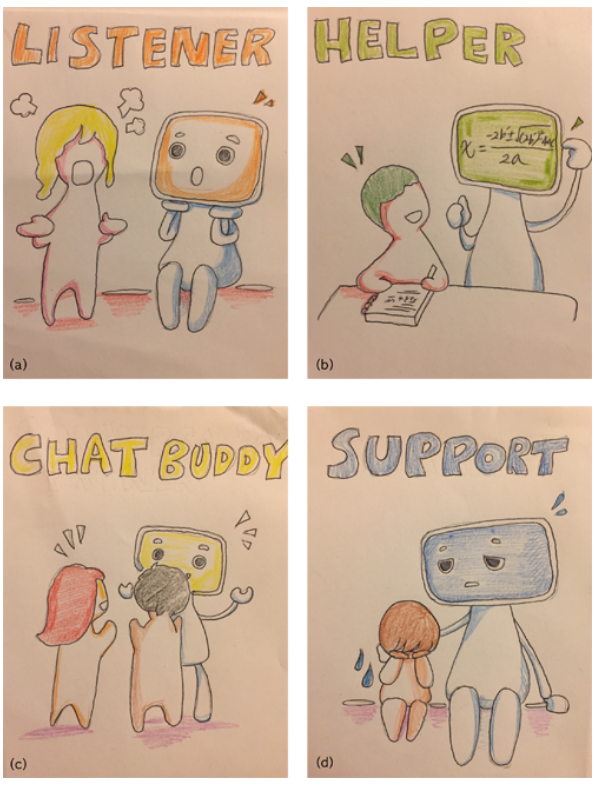

Figure 4: Samples of robot attribute cards. 'Ideal' characteristics of social robots that teens mentioned in sticky notes.
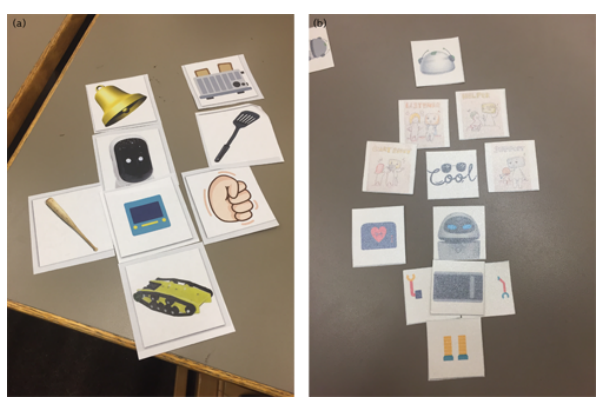

Figure 5: Two examples of robots created in the virtual reality card game.

Teens played the card game to use robot parts to create a stress-reducing robot. cards that teens mentioned in the previous stage (Figure 4). For collaboration, we prepared each of the game cards and a set of instructions for small groups (2-5) to try out and give feedback.

Nine teens in a club attended to the card game session. We separated them into two groups of 4-5 players and gave one deck of cards to each group. Teens were asked to assemble cards together to design a complete robot, just as they would do in the eventual VR game (Figure 5). Playtime was limited to five minutes for fast rotations. After finishing the session, we asked participants to complete a teen-friendly version of a usability questionnaire.

Teens expressed their enjoyment in customizing the robot. The average likeability for game playing 4.11 out of $5(n=9, S D=0.78)$. For the robot design, teens gave the lowest ratings, 3 out of $5(n=8$, $S D=1.51$ ), to the realistic feature while the highest ratings was given to the colored feature, 4.25 out of $5(\mathrm{n}=8, S D=0.46)$. One out of nine teens did not answer this one and the rest of open questions. Teens also wanted to remove cards with "human-like faces" because those cards were "creepy", and also the irrelevant accessories because they were "silly and not useful". Their preferred environments were 'cozy' places like classroom or nature. For characterization, all teens showed a strong preference for the robot attribute cards and wanted to include them to the robot design. For collaboration, teens indicated they enjoyed playing the game with their peers in open questions (e.g., "pretty good", "fun, innovative, interacted".) A few of teens explicitly mentioned their desires to play it with friends. We also observed two teens sitting next to each other were likely to naturally interact with each other the most; additional facilitation was needed to encourage all teens in a group to equally participate in.

Based on the teens' feedback from the card game session, we identified requirements for the VR game design. We decided to use the modularized robot parts, but to remove human-like faces and irrelevant accessories. We also decided to keep the idea of robot attributes for the future implementation. Finally, based on the the card game observation, we decided that two players would be ideal for eliciting smooth, focused collaboration.

\section{Design Stage 3: VR Game Development}

In the third stage, we developed a low-fidelity game in an immersive VR platform. Two environments, a classroom and an outdoor, and modularized robot parts in the card game were included in the VR environment for customization. For characterization, however, tobot attributes could not be included at this stage due to technical issues. Instead, we incorporated a few cartoon-like facial expressions into the robot head so that teens could adjust the 'character' of social robots.

For collaboration, We decided to use an asymmetric design [6] in order to promote focused communication between two teens. The inside player (the one using the VR headset) could assemble the robot parts upon the sphere-shaped robot frame. The outside player (the one using the desktop) could spawn robot parts for the inside player from the catalogue of 3D-modeled parts on the desktop screen (Figure 6). Throughout the game, players communicated via headsets and microphones. 


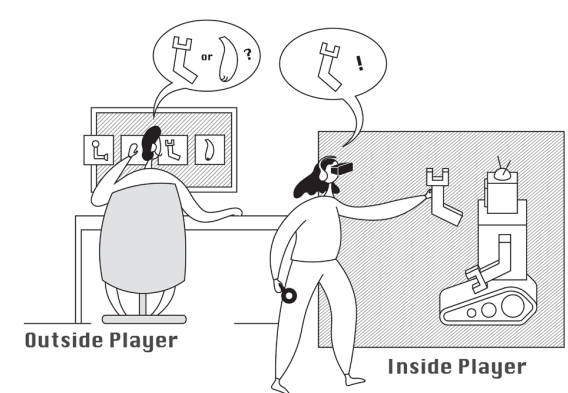

Figure 6: An illustration of asymmetric collaboration in VR.

The outside player asks preferred design of arms using the mic on headset, and spawn the inside player's choice in the VR space. The inside player picks up and adds the arm to the robot.



Figure 7: An assembled robot in the VR game.

The inside player assembled the robot in VR. The outside player used the catalogue of robot part options to collaborate in robot assembly.
We conducted a 45-minute usability testing session of our low-fidelity prototype with a club. Seven teens paired up and played the game by taking turns for each player position. At the end of the session, all seven teens had alternated into both roles as the inside and the outside player for the gameplay. The detail of the design environment and the 3D robot is in Figure 7.

Teens expressed their opinions on the VR game during and after the game play. We learned that teens (1) easily adapted to the asymmetric game design and enjoyed the collaboration in VR, (2) desired further and more elaborate interactions with the completed robot, and finally (3) wanted to share their robot designs with their family and friends, and in public spaces like the Internet.

\section{PRELIMINARY FINDINGS}

We learned that participatory HCD approach was effective to design a collaboration in a VR environment. By participating in our study, teens could see how their ideas, perspectives, and designs were addressed and integrated into each stage of the project. By seeing their suggestions (e.g., modularization of the robot parts) were directly applied to each step of design process, they were motivated to actively give and share feedback. Also, we observed that teens enjoyed not only the game but also the collaboration with peers. Once a robot was completed, they often cheered together and shared positive emotions from the accomplishment. During the gameplay, they willingly switched their roles to explore different aspects of collaboration without much disagreement or conflict.

Utilizing asymmetric design of our VR game seemed not only to enable easy prototyping of social robots, but also to encourage communications in teen-teen interactions. As each player had to rely on one other to complete the robot design, teens were expressive with one another and shared their design ideas aloud. Thus, it seemed that the asymmetric design amplified the teens momentary thoughts, opinions, and preferences, which could be important data for future studies.

\section{LIMITATION AND FUTURE DIRECTION}

Our design study is still in an exploratory level; the user feedback from the usability testing is preliminary. We plan to conduct further field studies to collect more structured data of teen-teen and teen-robot interactions as we make progress in the development. We also acknowledge the potential biases from respondents who were already highly interested in our project, VR or technology, and therefore revealed positive attitudes during the design process.

Despite the limitations, we recognize the effectiveness of a VR platform as not only a design tool, but also as an engaging collaborative platform for gathering interaction data from teens. Given this potential, we anticipate several future applications for the study of teen-teen and teen-robot interaction through VR collaboration. Moving forward, our next iterations of the game will continue to integrate ideas stemming from teens. 


\section{SELECTION AND PARTICIPATION OF CHILDREN}

This work included teens ages 14-17. Prior to conducting the current study, we obtained university IRB approval as well as school district research review. Upon introduction to the study, we made sure all participating teens read, understood, and maintained a copy of the consent/assent form. Any questions teens had were immediately answered by researchers. The consent/assent form included research statement, study activities, risk/stress/discomfort, benefits of the study. The consent/assent form also stated that participation was voluntary and teens could disengage at any time. Teens were also given the option to not be included in photos or videos. All photos and videos were used only for research purposes.

\section{REFERENCES}

[1] Jeremy N Bailenson, Nick Yee, Jim Blascovich, Andrew C Beall, Nicole Lundblad, and Michael Jin. 2008. The use of immersive virtual reality in the learning sciences: Digital transformations of teachers, students, and social context. The Journal of the Learning Sciences 17, 1 (2008), 102-141.

[2] Wilma A Bainbridge, Justin W Hart, Elizabeth S Kim, and Brian Scassellati. 2011. The benefits of interactions with physically present robots over video-displayed agents. International Journal of Social Robotics 3, 1 (2011), 41-52.

[3] Jim Blascovich and Jeremy Bailenson. 2005. Immersive virtual environments and education simulations. (2005).

[4] Simon Bowen, Helena Sustar, Daniel Wolstenholme, and Andy Dearden. 2013. Engaging teenagers productively in service design. International journal of child-computer interaction 1, 3-4 (2013), 71-81.

[5] Cynthia L Breazeal. 2004. Designing sociable robots. MIT press.

[6] Damien Clergeaud, Joan Sol Roo, Martin Hachet, and Pascal Guitton. 2017. Towards seamless interaction between physical and virtual locations for asymmetric collaboration. In Proceedings of the 23rd ACM Symposium on Virtual Reality Software and Technology. ACM, 17.

[7] Chris Dede. 2009. Immersive interfaces for engagement and learning. science 323, 5910 (2009), 66-69.

[8] Scott W Greenwald, Wiley Corning, and Pattie Maes. 2017. Multi-User Framework for Collaboration and Co-Creation in Virtual Reality. 12th International Conference on Computer Supported Collaborative Learning (CSCL).

[9] Yuta Katsumi, Suhkyung Kim, Keen Sung, Florin Dolcos, and Sanda Dolcos. 2017. When nonverbal greetings âĂlJmake it or break itâĂi: the role of ethnicity and gender in the effect of handshake on social appraisals. Journal of Nonverbal Behavior 41, 4 (2017), 345-365.

[10] Jérémy Lacoche, Nico Pallamin, Thomas Boggini, and Jérôme Royan. 2017. Collaborators awareness for user cohabitation in co-located collaborative virtual environments. In Proceedings of the 23rd ACM Symposium on Virtual Reality Software and Technology. ACM, 15.

[11] Albert Mehrabian. 1968. Relationship of attitude to seated posture, orientation, and distance. Journal of personality and social psychology 10, 1 (1968), 26.

[12] Maria V Sanchez-Vives and Mel Slater. 2005. From presence to consciousness through virtual reality. Nature Reviews Neuroscience 6, 4 (2005), 332.

[13] William R Sherman and Alan B Craig. 2018. Understanding virtual reality: interface, application, and design. Morgan Kaufmann.

[14] Helena Sustar, Simon Bowen, Andy Dearden, Mark Fisher, and Dan Wolstenholme. 2013. Using popular culture to enable health service co-design with young people. $E A D(2013)$. 